\title{
ESTUDO DA CINÉTICA DE SECAGEM E ALTERAÇÕES FISÍCO-QUÍMICAS DO TOMATE (Lycopersicon esculentum)
}

\author{
R.G.SANTOS ${ }^{1 *}$, A.R.S.TELES ${ }^{2}$, A.M.CONCEIÇÃO², N.F.P. BARBOSA ${ }^{2}$ \\ ,G.F.SILVA ${ }^{3}$, L.F.MONTEIRO ${ }^{4}$ \\ ${ }^{1}$ Universidade Federal de Sergipe, Departamento de Engenharia Química \\ ${ }^{2}$ Universidade Federal de Sergipe, Departamento de Tecnologia de Alimentos \\ ${ }^{3}$ Universidade Federal de Sergipe, Laboratório de Tecnologias Alternativas \\ ${ }^{4}$ Universidade Federal de Sergipe, Departamento de Engenharia de Produção \\ *e-mail: renatayorana@hotmail.com
}

\begin{abstract}
RESUMO
O tomate é uma das frutas mais consumidas no mundo, sendo empregado tanto para o consumo in natura como para a obtenção de diversos produtos, dentre eles o tomate seco. A secagem consiste na remoção dos componentes voláteis presentes do alimento, este processo pode ser traduzido em linguagem matemática por meio de modelos semiteóricos.A difusividade efetiva (Def) é a facilidade com que a água é removida do material. O processo de secagem acarreta em uma série de alterações dos parâmetros físico-químicos. Os objetivos deste trabalho consistiram em avaliar a cinética de secagem do tomate (Lycorpesicon esculentum), o modelo semiteórico que melhor se ajusta, calcular a difusividade efetiva (Def) e avaliar por meio de analises físico-químicas as alterações decorrentes do processo de secagem de: $\mathrm{pH}$, Acidez titulável, ${ }^{\circ}$ Brix, cinzas, cor, umidade e teor de licopeno em base seca. O modelo de Midilli foi o que obteve um melhor ajuste para esta fruta, a difusividade efetiva calculada pela equação de Crank (1975) foi de 5,55*10 $0^{-9} \frac{\mathrm{m}^{2}}{\mathrm{~s}}$, o teor de umidade do tomate seco foi $9,25 \%$, pH de 4,39, Acidez titulável de $2,88 \%$, ${ }^{\circ}$ Brix de 3,1 , Cinzas de $9,41 \%$, teor de licopeno em base seca de $54,49 \mathrm{mg} / 100 \mathrm{~g}$ e apresentou como parâmetros de cor (a* 21,8, b* 15,6, C* 26,8, L 20,1; H 35,6).
\end{abstract}

Palavras Chave: Secagem, Tomate, Modelos e Alterações Físico-químicas.

\section{INTRODUÇÃO}

O tomate é uma das hortaliças mais consumidas em todo o mundo, sendo consumido tanto da forma in natura e em produtos processados tais como: molhos, sucos, sopas, Ketchup e tomate secos (MURATORE et al., 2008).

Grande parte dos legumes é bastante perecível, com vida útil limitada quando estes são mantidos sob temperatura ambiente (DURIGON et al., 2013). Este fato, juntamente com controle inadequado da qualidade inicial, incidência e gravidade de lesão, a exposição à temperatura inadequada, atraso indevido entre colheita e o consumo, acarretam em perdas significativas na qualidade de frutas como o tomate (RAUPP etal., 2009; EVRANUZ, 2011).O fruto in natura tem vida de prateleira de aproximadamente uma semana (SANINO et al., 2003).Logo por ser uma hortaliça altamente perecível e com ótima compatibilidade ao processo de desidratação, o tomate vem sendo apontado como uma das principais alternativas para reduzir as perdas e agregar valor à matéria-prima (ALMEIDA JÚNIOR et al., 2003). Além de o tomate seco apresentar-se como uma alternativa para o aproveitamento do excedente de produção 
disponibiliza ao consumidor, um produto sensorialmente diferenciado e que, por ser menos perecível, pode ser comercializado em qualquer período do ano (NACHTIGALL et al., 2000).

A secagem de produtos com elevado teor de umidade inicial apresenta diversas vantagens tais como: inibição da ação de microrganismos, manutenção de constituintes minerais, redução de custos de transporte, manuseio e estocagem, tornando-se uma alternativa para a solução dos problemas de perda, descarte e poluição; além disso, no caso particular do tomate a secagem promove, entre outras, alteração do sabor e textura do fruto, conferindo-lhe paladar exótico, muito apreciado pelos consumidores.

Os principais componentes da cor no tomate são o licopeno e o beta-caroteno (GOULD, 1991).

Em termos de pesquisa, vem-se observando nos últimos anos, interesse crescente pela qualidade deste produto. Estudos são desenvolvidos visando à investigação dos parâmetros de secagem e aplicação de novas tecnologias que minimizem os danos causados pelo calor à cor, textura, ao sabor e perda de nutrientes (TOTOBESOLA-BRABIER et al., 2002; VEJA et al., 2001; CAMARGO et al., 2004; 2005).

O comportamento de secagem de tomate é igualmente descrita em termos de correlações empíricas (DOYMAZ, 2007; MOVAGHARNEJAD; NIKZAD， 2007; SACILIK, 2007; SANJINEZ-ARGADOÑA, 2011; PURKAYASTHA et al., 2011; DEMIRAY; TULEK, 2012).

As propriedades físicas e químicas são modificadas dos alimentos, devido a alterações no teor de água da superfície e no centro do alimento ocorrem em diferentes velocidades ao longo da secagem (JANGAM; MUJUMBAR; LEI, 2010; CRUZ; BRAGA; GRANDI, 2012).
Os objetivos do trabalho foram a caracterização da cinética de secagem de tomate (Lycorpesicon esculentum), determinação do valor da difusividade efetiva (Def) e, além disso, avaliar as alterações físico -químicas decorrentes do processo de secagem.

\section{MATERIAIS E MÉTODOS}

\subsection{Matéria Prima}

Os experimentos foram realizados no Laboratório de Tecnologias Alternativas da Universidade Federal de Sergipe, campus de São Cristóvão - SE.

$\mathrm{O}$ tomate foi adquirido em comercio local. Os frutos foram selecionados de acordo com a qualidade, uniformidade e grau de maturação.

Os frutos selecionados foram lavados em água de molho em solução de hipoclorito de sódio a 200ppm por 15 minutos. Após a sanitização os frutos foram cortados em formato de placas para aumento da superfície de contato e trinta fatias foram utilizadas para medidas de sua espessura com parquímetro, posteriormente os demais foram colocados nas bandejas para secagem em secador de convectivo- PARDAL mod. PE 100.

\subsection{Secagem}

Para determinação da curva de secagem foi utilizado um secador convectivo PARDAL mod. PE 100 a $70^{\circ} \mathrm{C}$. As bandejas foram pesadas em intervalos de 20minutos ate atingir peso constante.

Os dados de secagem foram ajustados com os modelos da Tabela 1 estas equações matemáticas foram empregadas como correlações empíricas para descrever o comportamento de secagem. Estas equações expressam a razão de umidade (MR) em função do tempo. 
Tabela 1- Os modelos matemáticos aplicados à curva de secagem do tomate.

\begin{tabular}{cc}
\hline Nome do Modelo & Equação \\
\hline Newton & $M R=\exp (-k t)$ \\
Page & $M R=\exp \left(-k t^{n}\right)$ \\
Midilli & $M R=\exp \left(-k t^{n}\right)+b t$ \\
& \\
\hline
\end{tabular}

Fonte: DURIGON (2013)

Onde MR é dado por:

$M R=\frac{M-M e}{M 0-M e}$

Os ajustes dos modelos aos dados experimentais de secagem foram realizados mediante procedimento de regressão não linear do pacote Statistica (StatSoft, versão 7, Tulsa, EUA). O critério de escolha dos melhores ajustes se baseou na determinação do coeficiente de regressão $\left(R^{2}\right)$ (BARROS NETO; SCARMINIO; BRUNS, 1995), gerado pelo próprio software estatístico e do valor de outro critério, que foi o qui-quadrado reduzido $\left(\chi^{2}\right)$, que levam em consideração as respostas observadas experimentalmente e os valores preditos pelo modelo (DEMIR et al., 2004; DOYMAZ; ISMAIL, 2010), como mostrado na Equação (2)

$\chi^{2}=\frac{\sum_{i=1}^{N}(\text { MRexp }, i-M R \operatorname{Rrev}, i)^{2}}{N-Z}$

Foi considerado que as fatias de tomate apresentavam geometria de placa plana, devido esta consideração pode-se fazer uso da Equação 3 proposta por Crank (1975), onde esta equação é empregada para a forma geométrica de placa plana. A difusividade efetiva (Def) foi calculada pela plotagem dos dados experimentais em termos de $(-\ln (\mathrm{MR}))$ em função do tempo de secagem (CRANK, 1975). O gráfico de $(-\ln (\mathrm{MR}))$ versus o tempo de secagem (Figura 1) dá uma linha reta com inclinação dada pela Equação 3.
Figura 1. Gráfico de $(-\ln (\mathrm{MR}))$ Versus tempo de secagem.

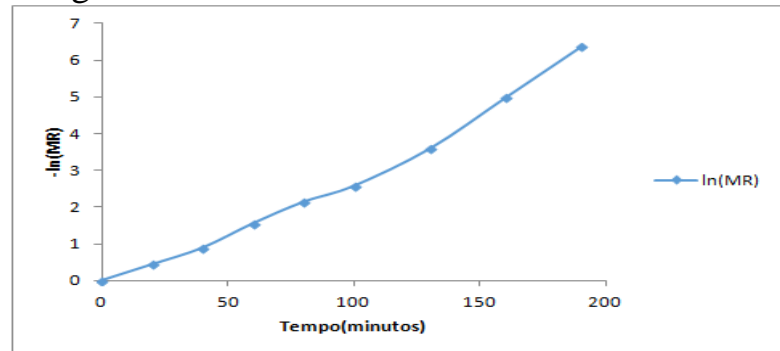

Fonte: Autor (2015)

Inclinação $=\frac{\pi^{2} * D e f}{4 * L 0^{2}}$

\subsection{Análises Físico-químicas}

O teor de umidade foi determinado pelo método de secagem em estufa para esterilização e secagem- BIOPAR a $70{ }^{\circ} \mathrm{C}$, determinado pela metodologia recomendada pelo Instituto Adolf Lutz (2008). O teor de sólidos solúveis, expresso em ${ }^{\circ} \mathrm{BRIX}$ foi determinado em refratômetro digital portátil KRUSS mod. DR201-95 de acordo com metodologia específica (CECCHI, 2003). A acidez foi obtida de acordo com as Normas Analíticas do Instituto Adolf Lutz (2008). O $\mathrm{pH}$ foi medido por meio do pHmetro HANNA mod. PH-21.O licopeno foi determinado por espectrofotometria utilizando o espectrofotômetro Bel phatonics-SP 2000UV de acordo com a metodologia de Nagata e Yamashita (1992), onde o conteúdo de licopeno foi expresso em $\mathrm{mg} / 100 \mathrm{~g}$ e estimado conforme a Equação 4, a cor foi determinada em colorímetro - KONICA MINOLTA mod.CR-10, utilizando o sistema de cor CIE L* a* b*: c*- luminosidade (branco/preto), $\mathrm{a}^{*}$ - cromaticidade eixo verde/ vermelho e $b^{*}$ - cromaticidade eixo azul/amarelo.

Licopeno $(\mu \mathrm{g} / \mathrm{ml})=-0,0458 \mathrm{~A} 663+0,204 \mathrm{~A} 645$ $+0,372 \mathrm{~A} 505-0,0806 \mathrm{~A} 453$ 


\section{RESULTADOS E DISCUSSÃO}

O tomate apresentava umidade final de 9,25\% (b.u). A Figura 2 apresenta os dados da cinética de secagem experimental e simulado obtido no secador para o tomate cortado em formato de placa sob temperatura de $70^{\circ} \mathrm{C}$.

Figura 2. Valor experimental e predito por modelos da cinética de secagem do tomate cortados em fatias á $70^{\circ} \mathrm{C}$.

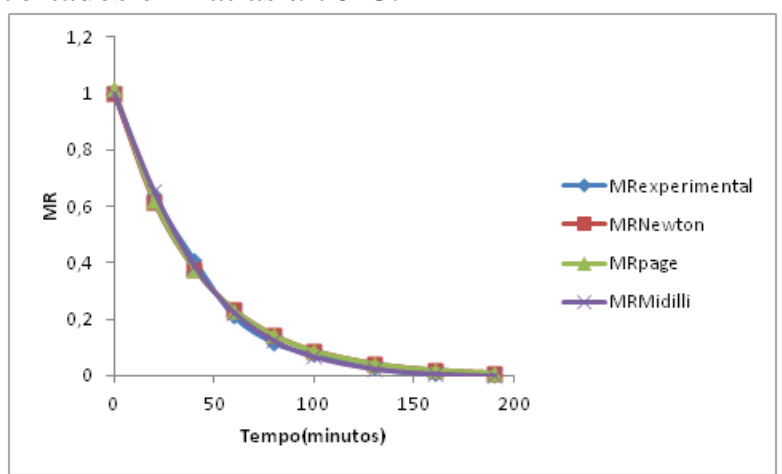

Fonte: Autor (2015)

O tempo necessário para secar o tomate cortado em fatias foi de 190minutos a uma temperatura de $70^{\circ} \mathrm{C}$. Esse resultado esta dentro do intervalo encontrado por RomeroPeña; Kieckbusch (2003), utilizando um secador de bandeja para secar tomate cortado o tempo de secagem obtido de 250 e 150 minutos a temperaturas de 60 e $80^{\circ} \mathrm{C}$, respectivamente.
Mas o tempo necessário para realização da secagem pode ser influenciado por uma serie de fatores tais como: o físico-químico, propriedades de material, a disposição geométrica do produto para a transferência de superfície de calor, velocidade e temperatura do ar, características do secador e tratamento anterior para secagem (DOYMAZ, 2007; HEREDIA; BARRERA; ANDRÉS, 2007; MURATORE et al., 2008; SANJINEZARGADOÑA et al, 2011.; DEMIRAY; TULEK, 2012).

Os dados experimentais da Figura 1 foram utilizados para selecionar $\mathrm{o}$ mais apropriado modelo para simular a cinética de secagem do tomate. A Tabela 2 mostra os valores de $\mathrm{R}^{2}$ e $\chi^{2}$ para os 3 modelos avaliados neste trabalho bem como os valores das constantes destes modelos .

A partir destes resultados, verifica-se que o melhor modelo de cinética para representar o processo de secagem de tomate é o modelo de Midilli descrito na Tabela 1 (MIDILLI et al., 2002) uma vez que este modelo apresentou o maior $\mathrm{R}^{2} \mathrm{e}$ o menor valor de $\chi^{2}$. Pois Quanto maior o valor de $\mathrm{R}^{2}$ e menor os valores $\chi^{2}$, melhor é a procedimento de ajuste (TOGRUL; PEHLIVAN, 2004; MAZUTTI et al, 2010; SANJINEZ-ARGANDOÑA et al., 2011).

Tabela 2. Constante, coeficientes de regressão e qui-quadrado reduzido dos modelos da Tabela 1.

\begin{tabular}{ccccccc}
\hline Nome do Modelo & $\mathbf{k}$ & $\mathbf{n}$ & $\mathbf{a}$ & $\mathbf{b}$ & $\mathbf{R}^{\mathbf{2}}$ & $\boldsymbol{\chi}^{\mathbf{2}}$ \\
\hline Newton & 0,024311 & - & - & - & 0,99814 & $4,43 \times 10^{-4}$ \\
Page & 0,024667 & 1,016 & - & - & 0,99830 & $4,05 \times 10^{-4}$ \\
Midilli & 0,014065 & 1,139 & - & $-0,000012$ & 0,99960 & $9,7 \times 10^{-5}$ \\
\hline
\end{tabular}

Fonte: Autor (2015)

A espessura média encontrado das fatias de tomate foi de $1,0 \mathrm{~cm}$. Os dados apresentaram desvio padrão de $0,05 \mathrm{~cm}$. Para o experimento, o valor de espessura médio foi considerado constante.
A difusidade efetiva do tomate cortado em fatias foi de $5,55 * 10^{-9} \frac{\mathrm{m}^{2}}{\mathrm{~s}}$ o resultado foi semelhante ao encontrado por Durigon et al (2013) que encontrou uma difusividade efetiva para os tomates cortados em quatro 
partes onde esta difusidade variou de $2,75 *$ $10^{-9} \frac{\mathrm{m}^{2}}{\mathrm{~s}}$ a $5,92 * 10^{-9} \frac{\mathrm{m}^{2}}{\mathrm{~s}}$.

As caracterizações físico-químicas do tomate seco e in natura encontram-se na Tabela 3.

Tabela 3. Caracterizações Físico-Químicas do Tomate.

\begin{tabular}{lll}
\hline Análises & Tomate seco & $\begin{array}{l}\text { Tomate in } \\
\text { natura }\end{array}$ \\
\hline $\mathrm{pH}$ & $4,39 \pm 0,01$ & $4,63 \pm 0,04$ \\
Acidez & $2,88 \pm 0,0023$ & $0,21 \pm 0,021$ \\
${ }^{\circ}$ Brix & $3,1 \pm 0$ & $3,2 \pm 0$ \\
$\begin{array}{l}\text { Licopeno } \\
\text { (b.s) }\end{array}$ & $54,49 \pm 0,01$ & $16,29 \pm 0,01$ \\
Umidade & $9,25 \pm 1,05$ & $96,44 \pm 1,20$ \\
Cinzas & $9,41 \pm 0,32$ & $0,41 \pm 0,013$ \\
\hline
\end{tabular}

Fonte: Autor (2015)

$\mathrm{O} \mathrm{pH}$ do tomate seco foi de $4,39 \pm 0,01$ e o do fruto in natura foi de 4,63 $\pm 0,04$,os valores encontrados estão bem próximo aos encontrados por Munhoz et al. (2011). O teor de sólidos solúveis ( ${ }^{\circ}$ Brix), não houve diferença entre o fruto seco e o in natura, entretanto está a baixo do encontrado por Silva et al.(2010), isto pode ter ocorrido devido ao grau de maturação dos tomates, variedades dos tomates, temperatura, tempo de secagem e a geometria do corte. Os valores de umidade na amostra in natura foi de 96,44 $\pm 1,20$ não diferindo do valor encontrado por Munhoz et al.(2011). Para a amostra desidratada a umidade encontrada foi de 9,25 $\pm 1,05$ este foi diferente do encontrado por Silva et al.(2010). Com relação ao teor de cinzas ocorreu um aumento significativo da amostra seca em relação ao in natura, devido à remoção da água presente acarretando no aumento deste parâmetro. A secagem acarretou em um acréscimo do conteúdo de licopeno devido ao processamento térmico, aumento este também constatado por outros autores (DEWANTO et al., 2002; HADLEY et al., 2002).O aumento de licopeno do tomate também ocorre devido à maior liberação da matriz celular quando os frutos são submetidos ao calor (TOOR; SAVAGE, 2006).

A Tabela 4 apresenta os valores médios da luminosidade (L), intensidade de vermelho $\left(a^{*}\right)$, intensidade de amarelo $\left(b^{*}\right)$, saturação da cor $\left(\mathrm{c}^{*}\right)$ e H (Ângulo hue) é o ângulo de tonalidade da cor que consiste de um valor expresso em graus das amostras de tomates secos e in natura.

Tabela 4. Coordenadas colorimétricas do tomate na forma in natura e seco.

\begin{tabular}{lcl}
\hline $\begin{array}{c}\text { Parâmetros } \\
\text { colorimétricos }\end{array}$ & $\begin{array}{c}\text { Tomate } \\
\text { seco }\end{array}$ & $\begin{array}{c}\text { Tomate } \text { in } \\
\text { natura }\end{array}$ \\
\hline $\mathrm{L}$ & $20,1 \pm 0,5$ & $21,3 \pm 1,0$ \\
$\mathrm{a}^{*}$ & $21,8 \pm 1,6$ & $10,26 \pm 1,9$ \\
$\mathrm{~b}^{*}$ & $15,6 \pm 0,9$ & $10,36 \pm 1,7$ \\
$\mathrm{c}^{*}$ & $26,8 \pm 1,8$ & $14,6 \pm 2,6$ \\
$\mathrm{H}$ & $35,6 \pm 0,5$ & $5,4 \pm 1,5$ \\
\hline
\end{tabular}

Fonte: Autor (2015)

Foi observado que houve uma pequena diferença da coordenada L nas duas amostras, as demais coordenadas apresentaram diferença em todos os parâmetros principalmente a coordenada $\mathrm{c}^{*}$ que determina a saturação da cor, isso pode ser associado à temperatura de secagem á $70^{\circ} \mathrm{C}$ que pode ter acarretado na degradação dos carotenóides.

\section{CONCLUSÃO}

O modelo que melhor se ajustou a curva de secagem do tomate foi o modelo de Midilli.

A difusividade efetiva encontrada para o tomate foi de $5,55 * 10^{-9} \frac{\mathrm{m}^{2}}{\mathrm{~s}}$. 
A secagem provocou alterações em todos os parâmetros físico-químicos analisados.

\section{NOMENCLATURA}

MR: Razão de umidade (adimensional);

$k$ : Constante de secagem $\left(\min ^{-1}\right)$;

$t$ : Tempo (min);

$n, \quad a, \quad b: \quad$ Constantes experimentais (adimensionais);

$e$ : expoente;

$\mathrm{M}$ : Massa no tempo $\mathrm{t}(\mathrm{g})$;

Me: Massa de equilíbrio(g);

M0: Massa inicial (g);

$\mathrm{R}^{2}$ : Coeficiente de regressão linear (adimensional)

$\chi^{2}$ : Qui-quadrado reduzido (adimensional)

MRexp, i e MRpred, i: razão umidade experimental e a prevista respectivamente (adimensional);

$\mathrm{N}$ : é o número de observações (adimensional);

Z: é o número de parâmetros (adimensional);

Inclinação: inclinação da reta $-\ln (\mathrm{MR})$ vs tempo $\left(\mathrm{min}^{-1}\right)$

L0: Metade da espessura médiada fatia do tomate $(\mathrm{m})$

Def : Difusividade efetiva $\left(\mathrm{m}^{2} \mathrm{~s}^{-1}\right)$;

Licopeno $(\mu \mathrm{g} / \mathrm{ml})$ : concentração de licopeno $(\mu \mathrm{g} / \mathrm{ml})$;

A663:Absorbância a 663nm;

A645:Absorbância a 645nm;

A505: Absorbância a 505nm;

A453:Absorbância a 453nm.

\section{REFERÊNCIAS}

ALMEIDA JÚNIOR, J. F.; SILVA, C. A. B.; SIQUEIRA, K. B. Produção de tomate seco em conserva. In: SILVA, C. A. B.; FERNANDES, A. R. Projetos de empreendimentos agroindustriais: produtos de origem vegetal. Viçosa: UFV, 2003. cap. 10, p. 397-417.
BARROS NETO, B.; SCARMINIO, I. S.; BRUNS, R. E. Planejamento e otimização de experimentos. Campinas: $\mathrm{Ed} . \mathrm{da}$ UNICAMP, 1995. 303 p.

Camargo, G. A. Novas tecnologias e prétratamentos: tomate seco embalado a vácuo. Campinas: UNICAMP, 2005. 162p. TeseDoutorado

Camargo, G. A.; Moretti, R. H.; Ledo, C. A. S. Quality of dried tomato pre-treated by osmotic dehydration, antioxidant application and addition of tomato concentrate. In: InternationalDryingSymposium, 14, 2004, São Paulo. Proceedings... Campinas: UNICAMP, 2004. p.2207.

CECCHI, H. M. Fundamentos teóricos e práticos em análise de alimentos, 2. ed. 166 Campinas: Unicamp, 2003.

CRANK, J. The mathematics of diffusion. 2. ed. Oxford: S.I. Claredon Press, 1975. $414 \mathrm{p}$.

CRUZ, P. M. F. da; BRAGA, G. C.; GRANDI, A. M. de. Composição química, cor e qualidade sensorial dotomate seco a diferentes temperaturas. Semina: CiênciasAgrárias, v. 33, n. 4, p. 1475-1486, 2012.

DEMIRAY, E.; TULEK, Y. Thin-layer drying of tomato (Lycopersicumesculentum Mill. cv. Rio Grande) slices in a convective hot air dryer. Heat and Mass Transfer, v. 48, n. 5, p. 841-847, 2012.

DEWANTO, V.; WU, X. Z.; ADOM, K. K.; LIU, R. H. Thermal processing enhances thnutritional value of tomatoes by increasing total antioxidant activity. Journal of 
Agricultural and Food Chemistry, Washington, v. 50,n. 10, p. 3010-3014, 2002.

DOYMAZ, I. Air-drying characteristics of tomatoes. Journal of Food Engineering, v. 78, n. 4, p. 1291-1297, 2007.

DURIGON, A.; MAZUTTI, M.A.; MOSSI, A.J.; TREICHEL. H. Effects of temperature on both drying kinetics and color of Italian tomato. Biochemistry and Biotechnology Reports.Jan./Jun., v.2, n.1, p. 1-12, 2013.

EVRANUZ, E. O. Drying vegetables: new technology, equipment and examples. In: Handbook of Vegetables and Vegetable Processing. (Ed.) Sinha, N. K. Blackwell Publishing Ltd, p. 299-315, 2011.

GOULD, W. A. Composition of tomatoes. In: Gould, W. A. (ed.). Tomato production, processing and quality evaluation. Connectitut: AVI Publishing Co., 1991. cap.2, p.344-358.

HADLEY, C. W.; MILLER, E. C.; SCHWARTZ, S.J.; CLINTON, S. K. Tomatoes, lycopene, and prostate cancer: progress and promise. Experimental Biology and Medicine, London, v. 227, n. 10, p. 869880, 2002.

HEREDIA, A.; BARRERA, C.; ANDRÉS, A. Drying of cherry tomato by a combination of different dehydration techniques. Comparasion of kinetics and others related properties. Journal of Food Engineering, v. 80, n. 1, p. 111-118, 2007.

INSTITUTO ADOLFO LUTZ. Normas Analíticas do Instituto Adolfo Lutz. v. 1: 178 Métodos químicos e físicos para análise de alimentos, 4. ed. São Paulo: IMESP, 2008. 179 p. 105-106.
JANGAM, S. V.; MUJUMDAR, A. S.; LAW, C. L. Drying of Foods, Vegetables and Fruits, v. 1, 2010.

MAZUTTI, M. A.; ZABOT, G.; BONI, G.; SKOVRONSKI, A.; OLIVEIRA, D.; DI LUCCIO, M.; OLIVEIRA, J. V.; RODRIGUES, M. I.; TREICHEL, H.; MAUGERI, F. Mathematical modeling of thin-layer drying of fermented and nonfermented sugarcane bagasse. Biomass and Bioenergy, v. 34, n. 5, p. 780-786, 2010.

MIDILLI, A.; KUCUK, H.; YAPAR, Z. A. New model for single-layer drying. Drying Technology, v. 20, n. 7, p.1503-1513, 2002.

MOVAGHARNEJAD, K.; NIKZAD, M. Modeling of tomato drying using artificial neural network. Computers and Electronics in Agriculture, v. 59, n. 1, p. 78-85, 2007.

MURATORE, G.; RIZZO, V.; LICCIARDELLO, F.; MACCARONE, E. Partial dehydration of cherry tomato at different temperature, and nutritional quality of the products. FoodChemistry, v. 111, n. 4, p. 887-891, 2008.

MUNHOZ ,C, L.; UMEBARA,T.; BRANCO, G,I.; ARGANDONA， S,J,E. Caracterização e Aceitabilidade de Tomate Seco. Revista Brasileira de Tecnologia, v. 05, n. 01, p. 252-262, 2011.

NACHTIGALL, A. M.; FONSECA, A. S.; MACHADO, M. R. G.; VENDRUSCOLO, C. T.; GULARTE, M. A. Desenvolvimento de tomate desidratado em conserva. In: CONGRESSO BRASILEIRO DE CIÊNCIA E TECNOLOGIA DE ALIMENTOS, 17., 2000, Fortaleza. Resumos... Fortaleza: SBCTA, 2000, v. 3, p. 11.88 . 
NAGATA, M.; YAMASHITA, I. Simple method for simultaneous determination of chlorophyll and carotenoids in tomato fruit. Japanese Society for FoodScience and Technology, Tokyo, v. 39, n. 10, p. 925-928, 1992.

RAUPP, D. S. da; GARDINGO, J. R.; SCHEBESKI, L. S. dos; AMADEU, C. A.; BORSATO, A. V. Processamento de tomate seco de diferentes cultivares. Acta Amazonica, v. 39, n. 2, p. 415-422, 2009.

ROMERO-PEÑA, L. M.; KIECKBUSCH, T. G. Influência de condições de secagem na qualidade de fatias de tomate. BrazilianJournalofFood Technology, v. 6, n. 1, p. 69-76, 2003.

RAUPP,da S, D.; GARDINGO,R,J ;; SCHEBESKI; dos $\mathrm{S}$; AMADEU'A $\mathrm{C}$.; BORSATO, V,A.Processamento de tomate seco de diferentes cultivares.

SANJINEZ-ARGANDOÑA, E. J.; BRANCO, I. G.; BITTENCOURT, T. U.; MUNHOZ, C; L; Influência da geometria e da temperatura na cinética de secagem de tomate (Lycopersicumesculentum). Ciência e Tecnologia deAlimentos, v. 31, n. 2, p. 308312, 2011.

SANINO, A.; Cortez, L.B., Mederos, B.T. Vida de prateleira do Tomate (Lycopersicumesculentum), variedade "Débora", submetido a diferentes condições de resfriamento. In Workshop de tomate. Perspectivas e Pesquisas, 2003, Campinas.

SILVA, L.K,V.; PINHEIRO, S.E.; DOMINGUES, F.A.M.; DE AQUINO, C.A.; FIGUEIREDO, A.E.; DA COSTA, C.M.J.; CONSTANT, L.B.P. Efeito da pressão osmótica no processamento e avaliação da vida de prateleira de tomate seco.
CiênciasAgrárias, Londrina, v. 31, n. 1, p. 5566, jan./mar. 2010.

TOGRUL, T.; PEHLIVAN, D. Modelling of thin layer drying kinetics of some fruits under open-air sun drying process. JournalofFoodEngineering, v. 65, n. 3, p. 413-425, 2004.

MUNHOZ ,C, L.; UMEBARA,T.; BRANCO, G,I.; ARGANDOÑA, S,J,E. Caracterização e Aceitabilidade de Tomate Seco. RevistaBrasileira de Tecnologia, v. 05, n. 01, p. 252-262, 2011.

TOOR, R. K.; SAVAGE, G. P. Effect of semi-drying on the antioxidant components of tomatoes. Food Chemistry, v. 94, p. 90-97, 2006.

TOTOBESOLA-BARBIER, M.; MAROUZÉ, C.; Giroux, F. A Triz - based creativity tool for food processing equipment design. The TRIZ Journal Article Archives, Out, 2002.

VEJA, M.; GÓNGORA, N.; BARBOSA, C. Advances in dehydration of foods. Journal of Food Engineering, Amsterdam, v.49, p.271289, 2001. 